\title{
Captures
}

Figures, théories et pratiques de l'imaginaire

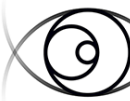

C A P T U R E S

Dîners imaginaires et repas fantasmes La recette de cuisine comme espace de projection littéraire

\section{Edwige Crucifix}

Volume 1, Number 2, 2016

Raconter l'aliment

URI: https://id.erudit.org/iderudit/1059811ar

DOI: https://doi.org/10.7202/1059811ar

See table of contents

Publisher(s)

Figura, Centre de recherche sur le texte et l'imaginaire

ISSN

2371-1930 (digital)

Explore this journal

Cite this article

Crucifix, E. (2016). Dîners imaginaires et repas fantasmes : la recette de cuisine comme espace de projection littéraire. Captures, 1(2).

https://doi.org/10.7202/1059811ar
Article abstract

Cet article se penche sur certaines particularités narratives de la recette de cuisine contemporaine (ici, le blogue de cuisine), envisagée comme un fantasme de lecture. Convoquant une sensualité de l'imaginaire à travers les indications techniques, la recette se fait espace de projection. La proximité narrative entre le blogue et la littérature gastronomique féminine de l'entre-deux-guerres invite aussi à interroger l'écriture de la recette comme pratique genrée, suggérant un potentiel contestataire.
Tous droits réservés (C) Edwige Crucifix, 2016

\section{(c) (i) $\Theta$}

This document is protected by copyright law. Use of the services of Erudit (including reproduction) is subject to its terms and conditions, which can be viewed online.

https://apropos.erudit.org/en/users/policy-on-use/ 
Version enrichie de cet article : http://revuecaptures.org/node/491

\title{
Dîners imaginaires et repas fantasmes
}

\section{La recette de cuisine comme espace de projection littéraire}

\section{Edwige Crucifix}

Résumé :

Cet article se penche sur certaines particularités narratives de la recette de cuisine contemporaine (ici, le blogue de cuisine), envisagée comme un fantasme de lecture. Convoquant une sensualité de l'imaginaire à travers les indications techniques, la recette se fait espace de projection. La proximité narrative entre le blogue et la littérature gastronomique féminine de l'entre-deux-guerres invite aussi à interroger l'écriture de la recette comme pratique genrée, suggérant un potentiel contestataire.

\author{
I/ est temps à \\ Il est temps à présent \\ Tandis que vous \\ Tandis que vous brassez \\ De glisser un présent \\ Pour votre fian \\ Pour votre fiancé \\ Un souhait d'amour \\ Un souhait d'amour s'impose \\ Tandis que la \\ Que la pâte repose \\ Graissez le plat de beurre \\ Et laissez cuire une \\ Et laissez cuire une heure
}

«Recette pour un cake d'amour », Jacques Demy1.

La « recette pour un cake d'amour » que Jacques Demy glisse dans son adaptation du conte de Perrault ne correspond certainement pas à l'idée qu'on se fait en général d'une recette de cuisine : elle est absolument non cuisinable. Il suffit pour s'en convaincre de revoir Catherine Deneuve, les manches de sa plus belle robe traînant dans la farine, aux prises avec les indications chantées par son double en guenilles. À l'image de cette chatoyante tenue domestique, les instructions qui servent à la préparation du cake d'amour sont une affaire 
d'apparat, lue par la souillon et exécutée par la princesse dans cette scène de dédoublement. Loin de se limiter à une liste d'indications numérotées, la recette de cuisine peut donc être déclamée comme un poème ou narrée comme une histoire et interprétée par des personnages. Sa fonction varie de même, suggérant une distinction de nature entre la recette de cuisine technique, celle qui a pour dessein d'être cuisinée, et cette recette d'apparence plus immédiatement littéraire, surtout lorsqu'elle figure dans un texte de fiction. Ainsi que le suggère Sarah Sceats, la recette sert alors par exemple à développer le caractère d'un personnage ou à rythmer l'intrigue et remplit souvent une fonction métaphorique en plus de sa fonction littérale (169).

En revanche, la recette technique, celle du livre de cuisine et du magazine féminin, se rangea priori près des fourneaux plutôt que sur les rayonnages des bibliothèques. Elle est un palier qui ne vaut que par sa capacité à la matérialisation, jugée à rebours depuis son résultat. Elle ne semble rien raconter au-delà de ce qu'elle dit explicitement. Comme tout discours, elle s'inscrit pourtant dans une situation énonciative, qui est, selon Susan Leonardi, une invitation à repenser son pouvoir narratif. Affaire de goût, elle est d'abord tributaire de l'imagination du lecteur, qu'il se mette effectivement aux fourneaux ou pas. Nous collectionnons en effet aussi les recettes à un autre dessein que celui de les cuisiner, peut-être pour les « goûter en esprit르 » (Wilson). Le repas promis par la recette quelle qu'elle soit est donc déjà, au-delà du plat qu'elle servira ou non à réaliser, un fantasme de lecture. Le langage de toute recette est performatif, en ce qu'il convoque une sensualité de l'imaginaire à travers les indications techniques. Il suggère aussi que la recette se prête comme support à d'autres désirs. Elle devient un espace de projection aussi bien pour le lecteur-cuisinier que pour son auteur.

Ce point commun entre la recette de cuisine technique et la recette de cuisine littéraire témoigne de la frontière poreuse entre la matérialité et la fiction, le physique et l'imaginaire, le sensuel et le poétique, qui n'est pas simplement une affaire de littérature. Alors que le discours alimentaire et les images de nourriture colonisent de plus en plus de domaines de notre vie quotidienne, la propension fantasmatique de l'aliment est continuellement exploitée par différents médias, que ce soit sous couvert de réalisme ou par l'entremise de la fiction, ainsi qu'en témoigne l'immense succès des émissions de télé-réalité, séries documentaires, chaînes télévisées, films et romans ayant pour thème principal la cuisine. Si le talent du publicitaire consiste à nous faire oublier que cette étourdissante prolifération d'images alléchantes s'adresse avant tout à nos appétits consuméristes, ces exemples prouvent assez le pouvoir fantasmatique que l'on fait jouer à l'aliment — et par extension, à la recette - au moins depuis la naissance de la littérature gastronomique.

Tout en considérant l'évolution de l'écriture de la recette à travers ce genre littéraire, notre article interroge plus spécifiquement le potentiel littéraire et fantasmatique de la recette de cuisine à travers ses nouvelles formes d'écriture, principalement celle le cas du blogue de cuisine. Face à l'immense variété de blogues de cuisine qui pullulent sur la toile, nous ne prétendons pas offrir un survol général de l'ensemble de cette production. Au 
contraire, à travers un nombre d'exemples restreint, nous avons choisi de nous intéresser plus particulièrement à quelques blogues à succès que nous avons identifiés par le nombre d'abonnés, la reconnaissance conférée par d'autres médias (magazines, chaînes de télévision, émissions de cuisine) ou par des prix, des interviews ou des classements. Comme le support numérique du blogue permet au lecteur de naviguer au hasard de son moteur de recherche et au gré des liens listés dans les marges des recettes, la consommation de la blogosphère culinaire ne se limite pas à une seule langue. Les exemples qui figurent ici sont donc francophones aussi bien qu'anglophones, suggérant une certaine unité de ton entre les blogues à succès audelà de la différence linguistique. Ces blogues partagent en effet une certaine teneur qui ne tient pas seulement à leur support numérique.

Le présent article avance en outre que l'écriture contemporaine de la recette est largement tributaire d'un retournement important, quoique peu remarqué, dans la tradition gastronomique : l'entrée des femmes dans ce genre littéraire durant la période de l'entre-deux-guerres. Sous la plume des pionnières de la littérature gastronomique féminine, l'écriture de la recette de cuisine acquiert en effet des caractéristiques que l'on retrouve à divers degrés aujourd'hui dans les blogues de cuisine à succès, dont la très grande majorité sont écrits par des femmes. En établissant un parallèle entre les mécanismes narratifs à l'œuvre dans la rédaction de ces blogues et les recettes proposées par les pionnières de la littérature gastronomique féminine, nous ne soulignons pas seulement une continuité littéraire d'une forme à l'autre, mais mettons en avant également un potentiel féministe inarticulé, commun à ces deux pratiques d'écriture. Sans être centrale, la question du genre reste donc toujours palpable. En effet, à travers ces deux pratiques, la recette de cuisine fonctionne non seulement comme le vecteur d'une projection sensuelle et imaginaire, mais également comme le moyen de créer une communauté littéraire féminine, et peut-être de formuler une émancipation du rituel domestique à travers sa mise en mots.

Diagnostiquer l'entrée des femmes dans la littérature gastronomique semble d'abord contre-intuitif pour un genre que l'on considère volontiers comme éminemment féminin. Pourtant la littérature gastronomique est d'abord une affaire d'hommes, ébauchée sous la plume de ses premiers théoriciens, bourgeois soucieux d'affirmer leur hégémonie sur le bon goût (Aron). De Brillat-Savarin à Escoffier, la gastronomie s'ébauche comme une affaire de professionnels, dont la pratique passe au cours des $\mathrm{XIX}$ et $\mathrm{XX}$ siècles des grandes maisons bourgeoises aux restaurants et autres palaces. Les femmes sont exclues de cet univers (certainement en tant que cuisinières et partiellement en tant que mangeuses) et confinées à l'expérience des cuisines familiales $\underline{3}$.

Il faut attendre l'entre-deux-guerres pour que les femmes fassent véritablement leur entrée dans la littérature gastronomique (McLean). La recette devient alors un objet littéraire à part entière, permettant souvent aux 
écrivaines de raconter leur sensualité et de partager leur expérience du foyer. L'œuvre de M.F.K. Fisher, qui constitue pour Leonardi une meilleure formation pratique que n'importe quel livre de cuisine technique (343), cristallise ce bouleversement stylistique. À travers une œuvre centrée autour de la cuisine, Fisher systématise l'ouverture de la littérature gastronomique à un nouvel espace et à un nouveau public, un mouvement commun à la littérature gastronomique américaine et française, qui entretiennent d'ailleurs un lien essentiel (Parkhurst Ferguson, 2014). Par l'écriture de la cuisine, l'œuvre de Fisher propose de reconsidérer le potentiel psychologique et littéraire de l'expérience gustative. Largement influencée en cela par les premiers théoriciens de la gastronomie française, elle s'en distingue néanmoins en faisant la part belle à l'expérience domestique et en ancrant de ce fait ses narrations gustatives dans sa propre expérience, donnant au genre une teneur plus immédiatement autobiographique.

Or cette caractéristique distingue aujourd'hui de nombreux blogues culinaires à succès. Les six finalistes sélectionnés dans la catégorie des blogues les mieux écrits pour le prix du magazine SAVEUR en 2015 (le SAVEUR Blog Award), l'un des plus prestigieux en son genre, constituent de bons exemples. Chacun de ces blogues est l'œuvre d'une femme et déclare un intérêt prononcé pour l'art de se raconter soi-même - ce qui est peu surprenant étant donné le format, qui se présente comme un journal intime numérique - mais aussi, pour le plaisir de raconter des histoires. Molly Wizenberg, l'auteure d'Orangette, se rêve ainsi en pionnière lorsqu'elle malaxe du pain (entrée du 3 février 2005), Phyllis Grant, l'auteure de Dash and Bella, a pour vocation de « raconter des histoires universelles 4 » et Cara Nicoletti, auteure de Yummy Books, pousse le vice jusqu'à ne partager que des recettes de fiction, comme si l'écriture de la recette était le prolongement du plaisir qu'elle a pris à les lire.

Les quelques recettes que Fisher livre dans The Gastronomical Me (1943) consacrent déjà ce projet littéraire en ce qu'elles lient toujours le plaisir gustatif à une jouissance de l'esprit, et souvent, de l'imaginaire. Évoquant les débuts de sa vie maritale à Dijon, elle se remémore notamment une certaine cassolette de chou-fleur dont son mari et leurs amis étaient alors friands. La même recette donne lieu à deux expériences très différentes : le souvenir d'un succès culinaire à Dijon contraste avec l'échec du plat quelques années plus tard, lorsqu'elle essaie de le reproduire en Californie : « Je pouvais, encore, concocter un bon plat... mais jamais aussi innocent, aussi simple... », conclut-elle avec nostalgie (103). Fisher attribue d'abord l'échec de cette reproduction à la qualité des ingrédients californiens, mais le partage de la recette se clôt sur le constat d'un manque plus sentimental : " Mais aussi, qu'était-il advenu de nos jeunes et simples appétits? ». Ces « appétits » à la fois physiologiques et métaphoriques apparaissent ainsi comme l'ingrédient essentiel, le liant qui manque à cette sauce blanche dont elle ne parvient pas à reproduire l'onctuosité passée.

Que les aliments, scènes de repas et même recettes servent de symboles, jouent avec l'imaginaire, il n'y a là 
rien de surprenant. Mais si la recette de la cassolette de chou-fleur de Fisher, comme celle de la miche de pain dont se souvient Wizenberg, racontent effectivement une histoire empreinte de nostalgie, elles sont plus qu'un conventionnel ubi sunt. Ces recettes servent aussi de vecteur au partage d'une expérience sensuelle en tissant une connexion entre le plaisir physique et le plaisir imaginaire, entre le corps et l'esprit. L'expérience gustative du souvenir (identifiée comme la motivation de l'écriture) et l'expérience de lecture se rencontrent et se répondent à travers les mots de la recette. Le goût ainsi recréé est à la fois celui du plat réel, disparu mais consommé par l'auteur, et celui de l'imaginaire du lecteur, celui du plat fantasmé qu'il se figure à travers les mots et probablement, la réminiscence de ses propres expériences culinaires. Bien qu'il s'agisse d'un souvenir, le plaisir provoqué par la narration de la recette n'appartient donc pas strictement au passé, mais plutôt au présent doux-amer de la nostalgie. La sensualité de toute recette résiderait donc, nous dit Bee Wilson, dans le présent de la lecture. La recette est en ce sens toujours fictionnelle parce qu'elle est toujours une projection. Wilson va plus loin que Wizenberg ou Nicoletti, affirmant même qu'elle préférerait ne jamais avoir à cuisiner ses recettes favorites pour ne pas gâter le goût parfait qu'elles ont dans son imagination. C'est d'ailleurs ce qui explique sa prédilection pour les recettes historiques comme celle des «Asperges en Sauce » du Cuisinier François, un livre de cuisine du XVIP siècle qu'elle découvre avec plaisir dans une anthologie publiée par William Sitwell en $2012 \underline{5}$. Le plaisir de cette recette démodée qu'elle n'a jamais essayée tient à son vocabulaire suranné et parfois incompréhensible, à la part de mystère nichée entre les indications techniques. Participant à l'engouement éditorial pour la cuisine d'antan et pour les produits du terroir, la fascination de Wilson est pourtant le contraire d'une véritable redécouverte. Au contraire, le plaisir repose sur un refus d'élucidation, qui sert de support au fantasme.

L'anthologie de recettes se soucie donc moins de traduire l'exactitude des indications que de les reproduire dans toute leur mystique : c'est la lecture qui est ici délectable, la recette étant consommée dès que le regard se pose sur la page. L'attention au visuel qui distingue les blogues de cuisine en vogue, bien davantage encore que le style d'écriture ou même que la faisabilité des recettes, signale le plaisir de cette consommation instantanée. Plus encore qu'un besoin de précision technique, l'image appétissante (et ici il s'agit presque toujours de photographies) invite moins à se mettre au fourneau qu'à faire dérouler les posts sur son écran. Le blogue s'offre lui-même à la consommation, il se dévore des yeux. C'est le cas de J'veux être bonne. récompensé par les magazines Elle et Glamour pour la beauté de ses photographies, qui signale la chose dès son titre : à travers la métaphore d'un vocabulaire outrancièrement genré faisant signe vers la concupiscence sexuelle, le blogue s'offre à la dévoration, annonce son ambition de séduire. L'auteure dit vouloir par ce biais faire « du bien aux yeux et au palais », attribuant la survivance du blogue à son goût pour la photographie (« $\mathrm{A}$ propos »). Stylistes ou photographes aussi bien que cuisinières, certaines auteures incluent d'ailleurs des précisions techniques quant au matériel utilisé pour les photos du blogue et des mises en garde contre 
I'utilisation non autorisée de leurs clichés. Aucune voix ne s'élève pourtant contre la reproductibilité des recettes, copiées-collées à outrance ou survolées sans être lues par un internaute plus spectateur que lecteur. À la différence d'autres sites dédiés à la nourriture, ces beaux blogues ne font néanmoins pas l'économie du mot, laissant ouverte la question du dialogue possible entre la recette-écrite et la recette-image, puisque grâce au déroulement infini de la page, la recette n'est plus tenue de se limiter au quota d'illustrations infligé au livre de cuisine. Aucun de ces éléments ne sert de clé interprétative à l'autre.

C'est ce que suggère aussi le Cookbook Book d'Annahita Kamali, qui reproduit simplement des photographies de différentes pages de livres de cuisine, d'origines et d'époques variées, sans annotation ni traductions, et dont l'intérêt repose sur la variété des formes et des formats. L'ouvrage s'apparente à un catalogue de design, faisant de la recette un bel objet, comme le montrent les deux pages reproduisant un petit livre de cuisine anonyme en japonais, dont les photographies de plats inconnus en noir et blanc garantissent le potentiel évocateur (174-175). La projection dépend ici d'une mise en abyme de la photographie et du livre (les photographies du livre original photographiées par Florian Böhm et le livre dans le livre). Si le festin est imaginaire, l'agrément est bien, en partie, sensuel. Le langage de la recette est en ce sens performatif, même lorsqu'il se passe de mots, détenant le pouvoir de susciter le plaisir gustatif - et tout ce qu'il implique de métaphorique - au-delà de la réalité éventuelle du plat à cuisiner. Ces recettes jouent effectivement avec les sens du lecteur, mais en créant une matérialité sans matérialité : la jouissance culinaire est suscitée sans qu'il soit besoin de se salir les mains, de suer au-dessus d'une casserole bouillante, de respirer les effluves déplaisants de la poubelle où s'entassent les rebus inévitables. Mais aussi sans manger, sans grossir, sans digérer, sans la médiation pesante du corps physiologique.

Cette mise à distance du corps sans négation du plaisir gustatif rappelle «Trente-huit, cinq » (Colette, 1986 [1932]), l'une des chroniques culinaires que Colette, autre pionnière de l'écriture gastronomique féminine, a écrites pour Vogue entre 1924 et 1932. L'article met en scène une narratrice malade qui ne peut rien avaler sans dégoût. Chargée cependant d'écrire la recette de la poitrine de bœuf à la languedocienne, elle oscille dans son délire fiévreux entre l'horreur que lui inspire la grasse réalité du plat et la douceur bienfaisante de nourritures plus délicates. Paradoxalement, elle répond à cet impératif rebutant par une substitution, livrant à la place sa recette du lait d'amandes, annoncée comme une « leçon d'élégance » : «Encore manquera-t-il, à ma recette, ce qui rend le lait d'amandes plus doux à l'âme, et aux lèvres : un ciel d'un bleu cendré, percé d'étoiles larges, embrumées par la végétale humidité d'un printemps marocain » (1986 [1932]: 91). Cet ingrédient insolite transforme le lait d'amande en nourriture spirituelle, plus douce encore aux lèvres qui parlent, qui murmurent les mots de la page, qu'à celles qui goûtent. Cette mise en abyme de l'écriture de la recette interroge sa fonction en questionnant la distinction entre l'autorité du « texte consultable » et la flexibilité du « texte interprétable $\underline{6}$ » (Rosello: 135). L'article se finit avec une autre recette liquide et odorante : celle du vin 
d'oranges. Et ici encore, le plaisir des sens est celui des mots de la recette, préférables même à l'ingestion, ainsi que le précise l'auteure : «n'ayez crainte, je ne le boirai pas, c'est assez de le flairer. Les yeux fermés, le nez ouvert, je relirai, pour Vogue, son histoire » (Colette, 1986 [1932]: 93). L’histoire en question, lue « les yeux fermés ", dégustée olfactivement, se raconte à travers cette recette finale, incarnation du paradoxe sensuel de cette courte chronique.

Mais qu'en est-il des plats cuisinés par les blogueuses dont les photographies trahissent assez l'encombrante matérialité? Face à l'avalanche de recettes qu'on trouve en ligne, le lecteur en oublie de penser à la consommation effective des plats. Ceux-ci semblent d'ailleurs n'avoir été préparés que pour se figer sur la surface brillante de l'écran. Et il est impossible qu'une telle abondance de mets disparaisse dans un seul estomac : la blogueuse ne saurait être gourmande que métaphoriquement. Si Colette manifeste certainement dans d'autres textes des appétits plus physiologiques, sa chronique met ainsi en lumière la capacité de la recette à libérer le plaisir gustatif de son enclave matérielle. Parade idéale pour bien d'autres jeunes femmes à la mode qui, à défaut de déguster de gras objets de convoitise alimentaire, les dévorent des yeux, les goûtent en rêve, en échangeant sur les réseaux sociaux des recettes qu'elles ne cuisineront jamais et d'alléchantes images de foodporn. À travers la lecture de la recette, la médiation esthétisante de l'imaginaire satisfait les appétits les plus désincarnés.

L'histoire racontée par la recette, au-delà de ses indications techniques, au-delà du plaisir gustatif et de sa fonction diégétique, assouvit en somme d'autres appétits que ceux du palais. Comme Barthes le suggère à propos des recettes du magazine Elle, celles-ci servent avant tout de support à l'imagination des lectrices et alimentent leurs rêves de bourgeoisie (1957: 121). L'imaginaire devient celui d'un repas-fantasme sur lequel la lectrice de recette projette un désir socio-économique. Cette « cuisine du revêtement et de l'alibi » (Barthes: 120) convoque la sensualité des lectrices du magazine, mais, à la différence des textes de Fisher ou de Colette, moins pour susciter son plaisir gustatif que pour combler en esprit ses rêves d'ascension sociale. La recette ne raconte pas seulement un plat, mais également un style de vie.

Bien des blogues culinaires exploitent ce potentiel relevé par Barthes, comme celui de la styliste et photographe Christelle Tanielian (2008-). En dépit de sa vocation déclarée, la recette n’apparaît qu'à la fin du billet, après avoir été plus ou moins racontée par Christelle dans une prose décomplexée. L'auteure s'avoue d'ailleurs souvent une imparfaite cuisinière et confesse même que son blogue de recettes est avant tout un moyen de s'adonner à sa passion pour le voyage et la photographie. Ses recettes sont une fenêtre sur sa vie de « fille cool » et permettent de s'y projeter, de voyager avec elle de son bel appartement de Montréal aux quatre coins de l'Europe. Les photographies qui les accompagnent ne sont pas seulement celles des plats mais aussi celles de paysages toscans, de ses amis cueillant des hortensias dans un immense jardin, de 
scènes de marché, de bougies trônant sur des couronnes de fleurs. La lecture de ses recettes convoque des rêves de voyage, de confort, de bon goût, de convivialité et de temps libre, des formes de luxe sans doute, mais moins immédiatement économiques. Christelle is flabbergasting n'est pas une référence que l'on consulte au moment de préparer le repas du soir; le blogue peut être suivi comme un feuilleton ayant la particularité de transporter son lecteur dans l'univers mode et déluré de l'auteure.

La tendance joue en effet un rôle non négligeable dans la propension fantasmatique de la recettæe. Des tendances alimentaires actuelles comme le véganisme, le régime paléo ou sans gluten, dont le succès n'est pas simplement imputable à la restriction physiologique ou à l'impératif moral, font aussi la promotion d'un style de vie $\underline{8}$. Même des effets de mode qui n'onta priori rien à voir avec la table influencent les habitudes alimentaires. L'inévitable tendance hipster, dont les barbes touffues et meubles en bois brut incarnent un fantasme d'authenticité, se traduit dans la littérature gastronomique en une nostalgie d'apparat pour le slow food, par une prédilection pour les légumes oubliés et les céréales biologiques, mais également par un vif intérêt pour la nourriture populaire et sans chichi, comme la cuisine de rue. Le rôle d'internet et de ses réseaux sociaux est central dans la diffusion de ces orientations alimentaires et met en avant l'importance de l'image ( en particulier le graphisme du site et le style de la photographie) dans l'établissement de ces fantasmes sociaux $\underline{9}$.

La recette en tant qu'acte littéraire imbriqué dans une situation énonciative est donc bien une mise en scène. Elle ébauche aussi un certain ethos narratif qui, en dépit de l'apparente transparence autobiographique, est lui aussi une construction fantasmatique. Le plaisir à lire la recette dépend également de ce «bavardage 10 » narratif (Leonardi: 343). En atteste peut-être l'immense succès de ces personnalités (aux deux sens du terme) que sont Philippe Etchebest, Jamie Oliver, Trish Deseine, Nigel Slater, Nigella Lawson ou Gordon Ramsay, mais aussi celui des auteures de blogues de cuisine de grande audience. Malgré leur apparence de journal intime, ces blogues sont aussi des curriculum vitae retraçant le parcours professionnel de leurs auteures, listant des moyens de les contacter, établissant une présence publique. L'accès au fantasme dépend d'une mise en scène impeccable de la vie des blogueuses, dont l'artificialité est masquée par la sensation d'intimité établie par la tonalité biographique, comme l'illustre le titre du blogue de Christelle Tanielian cité plus haut, Christelle is flabbergasting, qu'il faut certes prendre avec humour. Car la littérature gastronomique libère non seulement de l'effort, mais aussi de l'échec. Les prétentions d'honnêteté inhérentes à ces genres autobiographiques, de même que l'intimité créée par leur «bavardage », masquent le fait qu'ils ne sont que des vitrines travaillées, des portraits retouchés de leurs auteures, faisant la promotion d'un soi idéal et qui, tout en admettant avec humour leur propension à brûler les gâteaux, n'en offrent jamais le spectacle.

L'orientation genrée de ces autoportraits par la cuisine demande donc également qu'on évalue l'impact de 
cette forme narrative sur l'établissement d'un modèle de féminité. Les blogues ou mémoires de cuisinières dressent en effet, explicitement ou en creux, un portrait idéalisé de la femme en cuisine qui va de la traditionnelle « déesse domestique » — maman ou putain selon l'humeur de celui qu'elle sert 11 — à la baroudeuse de bon ton $\underline{12}$; deux modèles de femmes émancipées ayant réalisé leur accomplissement personnel par la cuisine. Écrire l'expérience domestique sous l'angle du plaisir peut alors servir à restituer à la ménagère une certaine agentivité - une caractéristique que l'on retrouve déjà sous la plume de Colette. Les élégantes lectrices de Vogue et de Marie-Claire pour lesquelles Colette écrit ses chroniques ne sont jamais complètement en rupture avec les ménagères plus âgées et moins élégantes que l'écrivaine met en scène, déesses rustres et rustiques du logis. Parmi ces femmes cuisinières, on trouve notamment Mme Yvon, « cordon bleu de grande race », qui n'est pour autant pas capable d'expliquer la recette de son bœuf à l'ancienne si ce n'est en répétant le nom de son ingrédient principal (Colette, 1986 [1932]:

83); pensons également à la concierge qui souffle à Colette la recette de son café au lait (2011: 14-16), un délice déjà mentionné dans Chéri (1920: 99). Les recettes qu'elle livre sont donc souvent héritées de tels personnages, transmises comme des mystères inexplicables, à l'instar du « poulet à la glaise ", secret d'une « gourmandise un peu brutale et préhistorique », comme le sont les femmes qui la lui ont transmise (1986 [1932]: 87). Et c'est justement par l'affirmation genrée de ce plaisir que la ménagère de Colette affirme son agentivité. Comme le suggère Susan Leonardi, l'écriture de la recette, motivée par le bonheur de partager une histoire personnelle ou collective, crée une communauté imaginaire à travers la transmission, le partage, l'échange de recettes qui transcende les barrières sociales, raciales et générationnelles (342-344).

L'intuition d'une communauté construite autour de la parole alimentaire est également la motivation scripturaire qu'invoque Kelsey Boyte, auteure du blogue Happyolks ${ }^{13}$. La communauté ainsi ébauchée autour d'une expérience partagée devient presque un idéal éthique, formulé depuis l'expérience individuelle de la narratrice. Grâce à l'acte d'écriture et au partage de la recette, la promulgation du plaisir culinaire domestique se charge même d'un potentiel contestataire, l'écriture de la recette permettant peut-être de déstabiliser les codes de cette activité honteusement traditionnelle. Certaines des blogueuses les plus populaires confient notamment avoir abandonné leur carrière professionnelle pour vivre leur « passion ». Pourtant, bien que le blogue soit un moyen pour certaines d'entre elles de gagner leur vie, elles ne le présentent pas comme une nouvelle carrière, mais plutôt comme l'emblème d'un renoncement à la vie active, que ce soit par goût (vient en tête l'exemple d'Edda Onorato, auteure de Un déjeuner de soleilh ou par nécessité. Le cas d'Anne Lataillade, auteure de Pupilles et Papilles, l'un des blogues cuisine en français les plus populaires (pas moins de 80000 visites par jour selon l'auteure) appartient à cette deuxième catégorie : contrainte d'abandonner sa carrière pour s'occuper de ses enfants, elle dit avoir commencé son blogue « sous peine d'attaquer Mr [sic] Papilles à grands coups de pic à glace en cas d'horaires tardifs, ou, pire encore, de [se] mettre au Sudoku! » (« $\mathrm{A}$ 
propos »). L'humour qui fait la succulence du blogue permet donc aussi de déstabiliser le stéréotype associé à la pratique domestique. Certes, la femme « retourne à sa place » dans l'organisation familiale; elle ne le fait toutefois pas sans porter un regard critique sur sa position. La nécessité domestique se transforme en projet par l'acte d'écriture.

Cependant, la difficulté d'émanciper l'écriture de recettes du poids des stéréotypes genrés signale une limite à la distanciation critique. La proclamation du plaisir de l'expérience domestique risque toujours d'apparaître comme la promulgation d'un idéal de féminité tristement conservateur. Les blogues pleins d'autodérision où des cuisinières déjantées réinventent la domesticité et la femme moderne, comme la Madame Chose de Geneviève Pettersen 14 , voisinent en effet presque indistinctement avec des portraits beaucoup plus lisses de ménagères épanouies dans leur rôle de femmes idéales. L'exemple préféré des internautes est sans doute Mimi Thorisson (plus de 230000 abonnés sur Instagram). Icône de perfection, Mimi est un personnage sans faille, une beauté cosmopolite ayant vécu à Paris et Hong Kong avant d'embrasser le rôle de fée du logis dans une demeure du Médoc. Sur les photographies de son blogue Manger, prises par son mari, l'élégante Mimi est vêtue à la dernière mode et sourit sans en faire trop, de la cuisine rustique où elle sublime le terroir local aux vignes où elle promène l'un de ses quatorze chiens. Ces images la mettent en scène seule vêtue d'un tablier débordant de pommes et pieds nus sur un antique carrelage, ou trônant debout à la table familiale, attirant les regards reconnaissants des sept enfants de la maisonnée, belle comme une peinture de la Renaissance. La vie de Mimi est d'autant plus propice au fantasme qu'elle est un choix : avant de vivre ce qu'elle décrit ellemême comme un « conte de fée $\underline{15}$ », Mimi a étudié la finance et travaillé pour CNN. Qui plus est, elle combine un équilibre décrit comme parfait entre dévouement familial et développement personnel (elle est présentatrice sur Cuisine+, écrit des livres, gère son blogue). Exprimant sa frustration face à cette mystique féminine excessivement conservatrice, la journaliste féministe Mona Chollet admet aussi son charme et attribue l'immense succès du blogue à sa capacité à transporter le lecteur loin de la laideur de sa vie quotidienne. Les recettes du blogue font de Mimi la gardienne d'une tradition perdue, un modèle de féminité moderne caractérisé par le choix conscient d'endosser (et de sublimer) un rôle traditionnel. Voilà la lectrice renvoyée au rôle de Peau d'âne, princesse cuisinière, l'humour de Demy en moins.

L'incursion croissante d'auteurs masculins dans le genre de la littérature gastronomique, qui pourrait susciter l'espoir d'un remaniement de ces fantasmes stéréotypés, semble en fait répondre tout autant à des modèles conservateurs. La cuisinière-artiste de Colette apparaît ainsi comme la contrepartie du cuisinier mis en scène par Julian Barnes dans Un Homme dans sa cuisine (2003), traduction qui ne rend pas l'ironie du titre original The Pedant in the Kitchen, mais qui insiste plutôt sur le genre de son narrateur-protagoniste. Ce «pédant » capable seulement de reproduire l'exactitude mathématique et rassurante de ses livres de recettes est le produit d'un discours genré : cuisinant pourtant chez lui, il ne peut se défaire de l'impératif de rigueur 
professionnelle incombant à son sexe. Il suggère ainsi une expérience de lecture antithétique à l'interprétation hédoniste qui conduit plus ou moins consciemment la littérature gastronomique de Brillat-Savarin à Nigella Lawson. La permanence d'une telle distinction sexuée au sein de la littérature gastronomique sous-tend la rupture entre professionnalisme et amateurisme et ce qu'elle implique de division entre les genres. Au lieu de marquer l'avancement des femmes dans l'espace des cuisines professionnelles, l'évolution de cette littérature suggère qu'au contraire, l'espace domestique devient lui-même un lieu d'affrontement potentiel entre les genres dont la cuisine est à la fois le champ de bataille et l'enjeu. Rêve de repas, repas de rêve, fantasme alimentaire ou identitaire, la recette, sous ses airs les plus innocents, raconte peut-être aussi cet affrontement. Surtout, tissant des histoires et des communautés, des liens entre les mots et les choses, entre l'estomac et l'esprit, elle déplace ces enjeux dans le domaine du verbe. L'enjeu hédoniste n'est plus seulement envisagé à travers la matérialisation du plaisir (l'objet alimentaire), mais aussi à travers la propension littéraire au plaisir, à travers le rapport entre le corps du lecteur et le corpus du texte imprimé ou numérique. La recette murmure ainsi tout bas, entre les lignes et les sifflements d'une cocotte, imaginaire ou réelle, littérale ou métaphorique, cette petite vérité domestique : le plaisir du mot ne supplante pas celui du mets, ils se confondent.

1. DEMY, Jacques (parolier) et Michel Legrand (compositeur). 2014 [1970]. « Recette pour un cake d'amour», Peau d'âne, version restaurée, réal. par Jacques Demy, prod. par Mag Bodard. France : Marianne Productions; Parc Film, DVD, 1:04:40-1:05:18.

2. [Nous traduisons.]

3. Concernant l'établissement du potentiel fantasmatique de la gastronomie bourgeoise, son rapport au genre et à l'identité nationale, consulter Parkhurst Ferguson (2004).

4. $\quad$ [Nous traduisons.]

5. L'anthologie de William Sitwell recommande d'ailleurs au lecteur de lire ces recettes pour elles-mêmes, sans en imaginer la concoction, devenue impossible.

6. $\quad$ [Nous traduisons.]

7. Parmi les blogues les plus populaires, on pourra penser à Minimalist Baker de Dana Shultz pour la cuisine végétarienne (environ 401000 abonnés Instagram et 16600 sur Twitter); pour la cuisine végane, à Deliciously Ella, blogue éponyme d'Ella Woodward (environ 955000 abonnés sur Instagram et 148000 sur Twitter); pour la cuisine paléo, à PaleOMG de Juli Bauer (environ 270000 abonnés sur Instagram et 27500 sur Twitter); pour la cuisine sans gluten, à Sassy Kitchen de Julia Gartland (19 625 abonnés sur Instagram); pour la cuisine « écocitoyenne », à Cuisine Campagne de Linda Louis. Les chiffres sont en date du $1{ }^{\text {er }}$ octobre 2016.

8. Le Wall Street Journal estime par exemple qu'aux États-Unis, bien que seulement $1 \%$ de la population soit intolérante au gluten, les produits sans gluten représentent un marché de 23 milliards de dollars (Jargon).

9. L'ampleur du phénomène peut se mesurer à l'importance de son public : plus de 5 millions d'abonnés pour le subreddit « food » (Anonyme, 2005-), qui recense des images de plats alléchants et près de 79 millions d'utilisations du marqueur «\#foodporn » sur Instagram (Systrom, 2016).

10. [Nous traduisons.]

11. Nigella Lawson, icône du foodporn, cristallise à elle seule ce paradoxe, offrant à la lectrice la possibilité d'être une « déesse domestique " ('expression est traduite de son titre How to be a Domestic Goddess), mais aussi une " salope », comme avec la recette de ses «slut's spaghetti » (sa traduction de pasta alla puttanesca) (Lawson, 2012).

12. Julie Andrieu représente un bon exemple de ce dernier archétype. De Fourchette et sac à dos aux Carnets de Julie. on voit la belle Julie, toujours impeccable dans son costume d'aventurière, lancer un regard séducteur à la caméra et goûter à l'exotisme du bout des lèvres. La découverte culinaire fait moins pour l'émission que le personnage de la présentatrice, modèle de féminité inoffensive, qui ne dérange personne et permet à la spectatrice de projeter ses fantasmes d'accomplissement personnel sans pour autant rompre avec un idéal traditionnel.

13. "My primary, and perhaps ONLY real mission with the blog is to create an environment where readers visit the site and leave feeling $[\ldots]$ encouraged that they do not journey their lives in isolation. [...] Together, we can be better, do better, and share in the 
beauty that is a full, sometimes messy life. » (Boyte citée par Anonyme, 2015.)

14. Geneviève Pettersen justifie en effet son projet éditorial en ces termes : « Parce que les histoires de bonnes femmes cachent toujours un fond de vérité, Madame Chose s'emploie à ce que cette sagesse populaire regagne la jeune femme moderne. [...] Madame Chose vous enseigne son art de vivre où récits, cataplasmes et recettes familiales se côtoient allègrement. 》 (20112014.)

15. [Nous traduisons.]

\section{Bibliographie}

Andrieu, Julie, Stéphane Jobert et Yann L'HÉnOREt. 2007 [-2012]. Fourchette et Sac à dos, diffusé sur France 5. Paris : Coyote; J. A. Productions.

Andrieu, Julie et Cédric Terrasson (réal.). 2012. Les Carnets de Julie, diffusion en cours sur France 3. Paris : Productions 3e CEil; J. A. Productions.

[s. a.]. 2005. «Food ». Reddit. <https://www.reddit.com/r/food/>.

[s. a.]. 2015. «2015 BLOG AWARDS: BEST WRITING FINALISTS. The 6 finalists for best writing in our 2015 SAVEUR Blog Awards ». Saveur, 7 avril. 丸ttps://www.saveur.com/gallery/2015-blog-awards-bestwriting-finalists>.

AOUAK, Hana. 2010. J'veux être bonne, en cours. «http://www.jveuxetrebonne.com>.

Aron, Jean-Paul. 1973. Le mangeur du XIXe siècle. Paris : Robert Laffont, 366 p.

BARNES, Julian. 2012. The Pedant in the Kitchen Bath : Shortlist, $136 \mathrm{p}$.

BARthes, Roland. 1957. Mythologies. Essais. Paris : Éditions du Seuil, 256 p.

Bauer, Juli. 2013. PaleOMG. <http://paleomg.com>.

Bonnier corporation. 1994. SAVEUR. <https://www.saveur.com>.

Borte, Kelsey. [s. d.] [-]. Happyolks. <http://happyolks.com>.

Chollet, Mona. 2015. Chez soi. Une odyssée de l'espace domestique. Paris : Zones, 250 p.

Colette, Sidonie-Gabrielle. 1920. Chéri. Rio de Janeiro : Americ-Edit, 235 p.

Colette, Sidonie-Gabrielle. 1986 [1932]. Prisons et Paradis. Paris : Fayard, 222 p.

COLETTE, Sidonie-Gabrielle. 2011. J'aime être gourmande. Paris : L'Herne, 97 p.

Demy, Jacques. 2014 [1970]. « Recette pour un cake d'amour », dans Peau d'âne, version restaurée, paroles de Jacques Demy, musique de Michel Legrand, produit par Mag. Bodard. France : Marianne Productions; Parc Film, DVD, 89 min.

Escoffier, Auguste et Pascal Ory. 2011. Souvenirs culinaires. Paris : Mercure de France, 248 p.

FISHER, Mary Frances K. 1989 [1943]. The Gastronomical Me. San Francisco : North Point, 252 p.

GarTLAND, Julia. 2014 [-]. Sassy Kitchen. <http://www.sassy-kitchen.com>.

Grant, Phyllis. 2015 [-]. Dash and Bella. $<$ http://dashandbella.blogspot.com>. 
JARGON, Julie. 2014. «The Gluten-Free Craze. Is It Healthy? ». The Wall Street Journal, 22 juin.

$<$ https://www.wsj.com/articles/how-we-eat-the-gluten-free-craze-is-it-healthy-1403491041>.

Kamalı, Annahita et Florian BöHm. 2014. Cookbook Book. Berlin : Phaidon, 320 p.

Lange, Frédéric. 1975. Manger, ou les jeux et les creux du plat Paris : Éditions du Seuil, 155 p.

LatallLade, Anne. 2005 [-]. Pupilles et Papilles. <https://www.papillesetpupilles.fr>.

Lawson, Nigella. 2001. How to Be a Domestic Goddess. Baking and the Art of Comfort Cooking New York : Hyperion, $374 \mathrm{p}$.

Lawson, Nigella. 2012. «Slut's Spaghetti ». The Home of Nigella Online. $<$ https://www.nigella.com/recipes/sluts-spaghetti>.

LEONARDI, Susan J. 1989. «Recipes for Reading. Summer Pasta, Lobster à La Riseholme, and Key Lime

Pie ». PMLA, vol. 104, no 3, p. 340-347.

Louls, Linda. 2006. Cuisine Campagne. $<$ https://www.cuisine-campagne.com>.

MérIGot (DE), Madame. 1976 [1794-1795]. «Diverses manières d'apprêter les Pommes de terre », dans La

Cuisinière républicaine, extrait d'un facsimilé. Cambridge : Schlesinger Library de Harvard, p. 16-17.

$<$ https://www.radcliffe.harvard.edu/sites/default/files/field image/library-blog/bastille6.jpg>.

McLean, Alice L. 2012. Aesthetic Pleasure in Twentieth-Century Women's Food Writing. The Innovative

Appetites of M.F.K. Fisher, Alice B. Toklas, and Elizabeth David. New York : Routledge, 195 p.

NicolettI, Cara. 2015 [2010]. Yummy Books. <http://yummy-books.com/about/>.

Onorato, Edda. 2009 [-]. Un déjeuner de soleil. <https://www.undejeunerdesoleil.com>.

Parkhurst Ferguson, Priscilla. 2004. Accounting for Taste. The Triumph of French Cuisine Chicago :

University of Chicago Press, 262 p.

Parkhurst Ferguson, Priscilla. 2014. Words of Mouth. What We Talk About When We Talk About Food

Berkeley : University of California Press, 296 p.

Pettersen, Geneviève. 2014 [2011]. Madame Chose. Guide du bien-vivre à l'usage de la jeune femme

moderne. $<$ thtp://archive.is/BXAls>.

Rosello, Mireille. 1996. Infiltrating Culture. Power and Identity in Contemporary Women's Writing

Manchester : Manchester University Press, 205 p.

SCEATS, Sarah. 2010. «The Use of Recipes in Contemporary Fiction», dans The Recipe Reader. Narratives,

Contexts, Traditions. Lincoln : University of Nebraska Press, p. 169-186.

SHuLTz, Dana. 2012 [-]. Minimalist Baker. <https://minimalistbaker.com>.

SitwelL, William. 2012. A History of Food in 100 Recipes Londres : Collins.

SYstrom, Kevin. 2010 [-]. «\#foodporn ». Instagram. <https://www.instagram.com/explore/tags/foodporn/?hl=fr$\underline{\mathrm{ca}}>$.

TANIELLIAN, Christelle. 2008 [-]. «Christelle Is Flabbergasting ».<http://christelleisflabbergasting.com>. 
THORISson, Mimi. 2012 [-]. Manger. <http://mimithorisson.com>.

WILSON, Bee. 2013. «The Pleasures of Reading Recipes». The New Yorker, 26 juillet. $<$ https://www.newyorker.com/books/page-turner/the-pleasures-of-reading-recipes >

WIZENBERG, Molly. 2004 [-]. Orangette. <http://orangette.net>.

WoOdWARD, Ella. 2012 [-]. Deliciously Ella. <https://deliciouslyella.com>. 